\title{
Resultado do tratamento da tuberculose com estreptomicina, isoniazida e etambutol (esquema SHM)
}

\author{
Pedro Dornelles Picon ${ }^{1}$, Maria de Lourdes Della Giustina ${ }^{1}$, Carlos Fernando Carvalho Rizzon ${ }^{1}$,

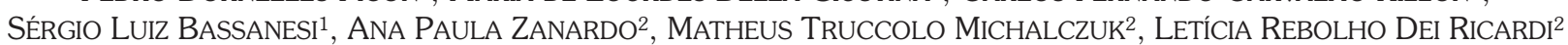

Objetivo: Avaliar o desempenho do esquema SHM (estreptomicina, isoniazida e etambutol), na rotina de trabalho de uma unidade ambulatorial de tratamento da tuberculose. Método: Setenta e oito pacientes tuberculosos, cujo tratamento prévio com o esquema RHZ (seis meses de rifampicina, isoniazida e pirazinamida) teve de ser interrompido devido a efeitos adversos, ou que não puderam receber o esquema $\mathrm{RHZ}$ por serem de alto risco para hepatotoxicidade a esse esquema, foram tratados ambulatorialmente com o esquema de 12 meses de SHM, de 1986 a 1994, em Porto Alegre, Rio Grande do Sul, Brasil. Resultados: Em três pacientes houve necessidade de troca de esquema por toxicidade (3,8\%). Nos 75 restantes observaram-se 58 curas $(77,3 \%)$, oito abandonos $(10,7 \%)$, cinco falências $(6,7 \%)$ e quatro óbitos $(5,3 \%)$. A taxa teórica de cura, que é o percentual de cura entre os baciliferos que fizeram tratamento regular, foi de 95,3\%. Reações adversas ocorreram em 32 pacientes (41\%), sendo as mais freqüentes as manifestações de dano vestibular, em 18 (23,1\%). Esses resultados foram comparados com os obtidos no mesmo ambulatório com o esquema de 12 meses de RHM (rifampicina, isoniazida e etambutol) e de seis meses de RHZ. Conclusão: O esquema SHM pode ser recomendado como alternativa para o tratamento da tuberculose quando o esquema RHZ não pode ser indicado. (J Pneumol 2002;28(4):187-192)

\section{Results of tuberculosis treatment with streptomycin, isoniazid, and ethambutol (scheme SHM)}

\begin{abstract}
Aim: To evaluate the performance of an SHM scheme (streptomycin, isoniazid, and ethambutol) in an outpatient clinic routine treatment for tuberculosis. Method: Seventy-eight patients with tuberculosis whose prior treatment with the RHZ scheme (six months of rifampicin, isoniazid, and pyrazinamide) had to be discontinued due to adverse effects, or who could not receive the RHZ scheme due to high risk for liver toxicity, were treated in the outpatient clinic with the 12 month SHM scheme from 1986 to 1994, in the city of Porto Alegre, Rio Grande do Sul, Brazil. Results: Three patients (3.8\%) required a scheme change due to toxicity. In the remaining 75 patients, 58 (77.3\%) were cured, eight (10.7\%) withdrew, five (6.7\%) presented failure, and four (5.3\%) died.

Theoretical cure rate, which is the percent of cure of patients who regularly followed the treatment, was 95.3\%. Adverse reactions were seen in 32 patients (41\%), the most frequent being vestibular damage in 18 patients (23.1\%). Results were compared to those obtained in the same outpatient clinic with the 12 month RHM scheme (rifampicin, isoniazid, and ethambutol), and with

the six month RHZ scheme. Conclusion: The SHM scheme may be recommended as an alternative for the treatment of tuberculosis whenever the RHZ scheme cannot be indicated.
\end{abstract}

\footnotetext{
* Trabalho realizado na Faculdade de Medicina da Universidade Federal do Rio Grande do Sul.

1. Médico da Secretaria Estadual da Saúde do Rio Grande do Sul, Brasil.

2. Acadêmico de Medicina.
}

Endereço para correspondência - Rua Filipinas, 295 - 94090-020 Porto Alegre, RS - Brasil. Tel. (51) 3340-0660; e-mail: pedpicon@ terra.com.br

Recebido para publicação em 24/7/00. Aprovado, após revisão, em 20/6/01.
Siglas e abreviaturas utilizadas neste trabalho

SHM - Estreptomicina, isoniazida e etambutol, respectivamente RHZ - Rifampicina, isoniazida e pirazinamida, respectivamente STH - Estreptomicina, tiacetazona e isoniazida, respectivamente $\mathrm{SPH}$ - Estreptomicina, ácido paraminossalicílico e isoniazida, respectivamente

RHM - Rifampicina, isoniazida e etambutol, respectivamente UT3 - Unidade de Tuberculose 3

TB - Tuberculose

$\mathrm{SRH}$ - Estreptomicina, rifampicina e isoniazida, respectivamente 
Descritores - Tuberculose. Estreptomicina. Isoniazida. Etambutol. Resultado de tratamento. Toxicidade. Antituberculosos.

Key words - Tuberculosis. Streptomycin. Isoniazid. Ethambutol. Treatment outcome. Antituberculosis agents. Toxicity.

\section{INTRODUÇ̃̃O}

A determinação da eficácia, eficiência e tolerabilidade de um esquema de antimicrobianos para o tratamento da tuberculose (tuberculostáticos) sempre foi uma preocupação dos Programas de Controle da Tuberculose antes de recomendá-lo como esquema padrão. Dessa forma, na busca do melhor resultado com o menor risco para o paciente, determinada associação de tuberculostáticos deve ser submetida a testes antes de ser indicada em grande escala.

No passado foram testados e utilizados esquemas de um ano, como o SPH (estreptomicina, PAS e isoniazida), o STH (estreptomicina, tiacetazona e hidrazida)(1) e o RHM

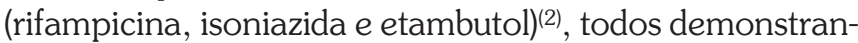
do resultados favoráveis. Os esquemas de curta duração, especialmente o RHZ (rifampicina, isoniazida e pirazinamida) ${ }^{(2-4)}$, também foram aprovados.

Por vezes, um esquema alternativo passa a ser indicado em situações especiais, como é o caso do que aconteceu no Estado do Rio Grande do Sul, Brasil. Nesse Estado, a padronização pelo Programa de Controle da Tuberculose do esquema RHM como primotratamento da tuberculose, em 1977, teve como conseqüência um incremento da taxa de hepatotoxicidade. Esta aumentou de 2,2\%, com o STH, para 7,3\%, com o RHM, nos pacientes internados no Hospital Sanatório Partenon (órgão de retaguarda do Programa)(5). Isso gerou a necessidade de buscar um esquema alternativo, chegando-se ao SHM (estreptomicina, isoniazida e etambutol)(6), que passou a ser utilizado nos casos de hepatotoxicidade pelo RHM e, mais tarde, nos casos de hepatotoxicidade pelo RHZ, quando este esquema substituiu o RHM no primotratamento da tuberculose, a partir de 1982. Com a utilização do RHZ, a taxa de hepatotoxicidade aumentou para $11,1 \%$ no mesmo hospital ${ }^{(5)}$.

Ainda no Hospital Sanatório Partenon, de 1982 a 1984, foram identificados como fatores de risco para hepatotoxicidade pelo RHZ: presença de tuberculose de disseminação hemática (TB miliar); mau estado clínico; sexo feminino; idade avançada. Em vista desses achados e também da observação de bons resultados do SHM em pacientes com hepatopatia medicamentosa, desde então, naquele hospital, passou-se a recomendar o esquema SHM, em vez do RHZ, nas seguintes situações: 1) mulheres com TB miliar e/ou em mau estado clínico; 2) homens com TB miliar e em mau estado clínico; e 3) pacientes tuberculosos portadores de doença hepática crônica(6).

Feitas essas considerações, os autores resolveram observar o desempenho do esquema SHM na rotina de trabalho de uma unidade ambulatorial de tratamento da tuberculose, na qual os critérios para indicação do esquema foram os acima referidos. Sabe-se que esse mesmo esquema foi utilizado no Estado de São Paulo na década de 1970, mas não foram encontrados registros bibliográficos dos resultados alcançados.

\section{MATERIAL E MÉTODOS}

Os autores elaboraram um protocolo de pesquisa, com informações padronizadas, para acompanhamento de pacientes ambulatoriais que recebessem o esquema SHM segundo os critérios de indicação citados acima. No período de janeiro de 1986 a dezembro de 1994, foram acompanhados todos os 78 pacientes que utilizaram SHM na Unidade de Tuberculose 3 (UT3), da Secretaria da Saúde do Estado do Rio Grande do Sul, Brasil. Os dados foram coletados por três pneumologistas treinados. Os aspectos éticos foram observados à medida que não houve intervenção dos pesquisadores nas decisões clínicas.

Entre os pacientes, havia 37 homens e 41 mulheres, todos em primotratamento. A média de idade foi 39 anos (de 15 a 79 anos), havendo 47 pacientes com 40 anos ou menos e $31 \mathrm{com}$ mais de 40 anos. Dezoito pacientes eram alcoolistas (23\%). Havia 58 pacientes com TB pulmonar (74,4\%), oito com TB miliar (10,2\%) e 12 com TB extrapulmonar (15,4\%). O diagnóstico foi confirmado em 71 pacientes (91\%), através de: baciloscopia positiva no escarro (60 casos); granuloma em material de biópsia (oito casos); baciloscopia positiva no lavado broncoalveolar (um caso); baciloscopia positiva em aspirado de lesão osteoarticular (um caso) e cultura do escarro positiva para $M$. tuberculosis (um caso). Nos outros sete pacientes (9\%) o diagnóstico foi feito com base nos achados clínicos e radiográficos e na presença de teste tuberculínico positivo.

O esquema SHM constituiu-se dos três tuberculostáticos (estreptomicina, isoniazida e etambutol) nos três primeiros meses, seguidos de isoniazida e etambutol por mais três meses e isoniazida por seis meses, totalizando 12 meses de tratamento.

O tratamento foi auto-administrado, com a medicação fornecida para 30 dias. A estreptomicina foi aplicada por via intramuscular, procurando-se observar sempre o mesmo horário. Os comprimidos de isoniazida e etambutol foram ingeridos após a principal refeição do dia, geralmente o almoço.

As doses recomendadas foram: estreptomicina, 750 a $1.000 \mathrm{mg} /$ dia (total entre 67,5 e $90 \mathrm{~g}$ ), para pacientes com 40 ou menos anos de idade, e 500 a $750 \mathrm{mg} /$ dia (total entre 45 e 67,5g), para aqueles com mais de 40 anos; 
isoniazida, 4 a $5 \mathrm{mg} / \mathrm{kg}$ de peso corporal ao dia; etambutol, 15 a $25 \mathrm{mg} / \mathrm{kg}$ de peso corporal ao dia.

Vinte e um pacientes receberam o esquema SHM desde o início devido a: doença hepática crônica (10 casos); TB miliar em mulheres (quatro casos); TB miliar em homens em mau estado clínico (três casos) e mulheres em mau estado clínico (quatro casos). Os demais 57 pacientes receberam o SHM em substituição ao esquema RHZ, utilizado por curto período de tempo, de cinco a 42 dias, com média de 13 dias, e interrompido devido aos seguintes efeitos adversos: hepatotoxicidade (45 casos); vômitos incoercíveis (11 casos); erupção cutânea (um caso). Os casos de hepatotoxicidade pelo RHZ foram diagnosticados com base na presença de sintomas sugestivos, surgidos após a introdução do esquema, e alteração de provas de função hepática (bilirrubinas $\geq 2,0 \mathrm{mg} \%$, ou transaminases $\geq$ três vezes o máximo valor normal).

Foi definido como uso regular da medicação sempre que o paciente comparecia na data aprazada para a consulta e informava estar usando corretamente os tuberculostáticos, inclusive especificando exatamente o número de comprimidos e a hora da tomada. Adicionalmente, seus familiares, quando argüidos, informavam ser o tratamento regular e a visitadora sanitária não detectava, no domicílio do paciente, sobra de comprimidos ou de frascos de estreptomicina. Todos os pacientes foram visitados no primeiro mês de tratamento, para confirmação do endereço e avaliação da regularidade. Nos casos de dúvida quanto à regularidade e nos casos de abandono do tratamento, visitas domiciliares foram realizadas mais de uma vez.

O controle do tratamento nos casos pulmonares bacilíferos foi realizado através da avaliação clínica, da baciloscopia mensal do escarro e pela radiografia simples de tórax no final do tratamento. Os pacientes com tuberculose na forma pulmonar e com baciloscopia inicialmente negativa realizaram, além disso, radiografia no final do segundo mês, quando era reavaliada a manutenção do esquema (avaliação do resultado do tratamento de prova). Os casos extrapulmonares eram acompanhados através de dados clínicos, exames radiológicos e de outros, quando necessários, de acordo com a localização da tuberculose.

Os eventos finais do tratamento foram:

Cura: pacientes que usaram os tuberculostáticos pelo tempo programado (12 meses), ocorrendo, nos casos bacilíferos, melhora clínica acompanhada de negativação do escarro até o 6ำ mês de tratamento, permanecendo assim até o final e, nos casos abacilíferos, melhora clínico-radiológica.

Abandono: pacientes que interromperam o tratamento antes de concluir o tempo programado, independente de sua situação clínica e/ou bacteriológica.
Falência: pacientes com persistência de baciloscopia positiva no escarro no $6^{\circ}$ mês de tratamento, ou que voltou a positivar, por dois meses consecutivos, após negativação inicial; ambas as situações acompanhadas de piora clínico-radiológica.

Óbito: pacientes que morreram durante o tempo programado de tratamento, motivado ou não por tuberculose.

Após a alta os pacientes foram seguidos através do sistema de informação do Programa de Controle da Tuberculose, que permite detectar o reingresso no programa em qualquer unidade de saúde do Estado.

A comparação das proporções observadas nos diferentes estratos foi realizada utilizando-se o teste do qui-quadrado, com correção de Yates sempre que necessário, e a comparação das médias observadas nos diferentes estratos foi feita através do teste $t$ de Student $e$ de análises de variância, aceitando-se um valor de $\mathrm{p}<0,05$ como de significância estatística.

\section{REsultados}

A Tabela 1 registra os eventos finais do tratamento em todos os 78 pacientes e em 68 casos, após a exclusão dos 10 com doença hepática crônica. Do total, três pacientes, que não eram portadores de doença hepática crônica, tiveram que trocar de esquema por toxicidade, restando 75 . Entre estes, 18 eram alcoolistas (24\%), sendo 13 entre os pacientes sem doença hepática crônica (20\%) e cinco entre os hepatopatas (50\%), diferença não significante $(\mathrm{p}=0,094)$.

Na Tabela 2 verifica-se que 59 pacientes $(78,7 \%)$ fizeram tratamento regular. Ao considerar apenas os 65 pacientes sem doença hepática crônica, a taxa de regularidade eleva-se para 84,6\%, possivelmente em decorrên-

TABELA 1

Eventos finais do tratamento com SHM no total de pacientes e nos pacientes sem doença hepática crônica. UT3, 1/1/86 a 31/12/94.

\begin{tabular}{lrrrrr}
\hline \multirow{2}{*}{ Eventos finais } & \multicolumn{2}{c}{$\begin{array}{c}\text { Todos os } \\
\text { pacientes }\end{array}$} & & \multicolumn{2}{c}{$\begin{array}{c}\text { Casos sem doença } \\
\text { hepática crônica }\end{array}$} \\
\cline { 2 - 3 } \cline { 5 - 6 } & $\mathbf{N}$ & $\mathbf{\%}$ & & $\mathbf{N}$ & \% \\
\hline Cura & 58 & 77,3 & & 53 & 81,5 \\
Abandono & 8 & 10,7 & & 7,7 \\
Falência & 5 & 6,7 & & 5 & 7,7 \\
Óbito & 4 & 5,3 & & 3,1 \\
Subtotal & 75 & 100 & & 65 & 100 \\
Troca de esquema & 3 & 3,8 & & 4,6 \\
Total & 78 & & & 68 & \\
\hline
\end{tabular}


TABELA 2

Eventos finais do tratamento em 75 pacientes* tratados com SHM, de acordo com o modo de uso da medicação. UT3, de 1/1/86 a 31/12/94.

\begin{tabular}{lccccc} 
Eventos finais & \multicolumn{4}{c}{ Modo de uso da medicação } \\
\cline { 2 - 3 } & \multicolumn{2}{c}{ Regular } & & \multicolumn{2}{c}{ Irregular } \\
\cline { 2 - 3 } \cline { 5 - 6 } & $\mathbf{N}$ & $\%$ & & $\mathbf{N}$ & $\%$ \\
\hline \multirow{2}{*}{ Cura } & 52 & 88,1 & & 6 & 37,5 \\
Abandono & 2 & 3,4 & & 6 & 37,5 \\
Falência & 2 & 3,4 & & 3 & 18,8 \\
Óbito & 3 & 5,1 & & 6,2 \\
Total & $59(78,7 \%)$ & 100 & & $16(21,3 \%)$ & 100 \\
\hline
\end{tabular}

* Excluídos os três casos de troca de esquema.

** ( ) percentual em relação ao total de pacientes.

\section{TABELA 3}

Efeitos adversos nos 78 pacientes tratados com SHM na UT3, de 1/1/86 a 31/12/94

\begin{tabular}{lrr}
\multicolumn{1}{c}{ Efeito adverso } & N & \% \\
\hline Vertigem & 19 & 24,4 \\
Digestivos & 11 & 14,1 \\
Erupção cutânea urticariforme & 3 & 3,8 \\
Neuropatia periférica & 3 & 3,8 \\
Acne & 3 & 3,8 \\
Zumbidos & 2 & 2,6 \\
Parestesia perioral & 1 & 1,3 \\
Hepatotoxicidade & 1 & 1,3 \\
Total & 43 & 55,1 \\
\hline
\end{tabular}

cia do menor número de alcoolistas, situação reconhecida como causa de não adesão.

Na presente série, a taxa de irregularidade de tratamento foi de 55,5\% nos alcoolistas e de $10,5 \%$ nos não alcoolistas, diferença significante $(p=0,0001)$. Por sua vez, a taxa de abandono de tratamento também foi maior e com significância estatística: $27,7 \%$ entre os alcoolistas e 5,3\% entre os não alcoolistas ( $p=0,023$ ).

Nos 78 casos estudados, 32 pacientes (41\%) apresentaram 43 efeitos adversos, cuja distribuição pode ser observada na Tabela 3. Vertigem foi o efeito adverso mais freqüente $(24,4 \%)$, tendo ocorrido em 10 pacientes com menos de 40 anos $(21,3 \%)$ e em nove naqueles com mais idade $(29 \%)(p=0,609)$. Em total de oito pacientes $(10,2 \%)$ houve modificação no esquema: três casos $(3,8 \%)$ de mudanças de esquema, dois por vertigem e um por hepatotoxicidade; dois casos $(2,6 \%)$ de suspensão da estreptomicina antes do prazo, ambos por vertigem; três casos $(3,8 \%)$ de redução da dose da estreptomicina $(2,6 \%)$, todos por vertigem.
TABELA 4

Eventos finais do SHM em 59 pacientes bacilíferos, de acordo com o modo de uso da medicação. UT3, de $1 / 1 / 86$ a 31/12/94.

\begin{tabular}{|c|c|c|c|c|c|c|}
\hline \multirow[t]{3}{*}{ Eventos finais } & \multicolumn{4}{|c|}{ Modo de uso da medicação } & \multicolumn{2}{|c|}{ Total } \\
\hline & \multicolumn{2}{|c|}{ Regular } & \multicolumn{2}{|c|}{ Irregular } & & \\
\hline & $\mathbf{N}$ & $\%$ & $\mathbf{N}$ & $\%$ & $\mathbf{N}$ & $\%$ \\
\hline Cura & 41 & 89,2 & 4 & 30,8 & 45 & 76,3 \\
\hline Abandono & 2 & 4,3 & 5 & 38,4 & 7 & 11,8 \\
\hline Falência & 2 & 4,3 & 3 & 23,1 & 5 & 8,5 \\
\hline Óbito & $1 *$ & 2,2 & $1^{* *}$ & 7,7 & 2 & 3,4 \\
\hline Total & 46 & 100 & 13 & 100 & 59 & 100 \\
\hline
\end{tabular}

* Óbito por lúpus eritematoso sistêmico.

** Óbito por AIDS.

Os pacientes receberam, em média, 18,6mg/kg de peso corporal de estreptomicina, $5,2 \mathrm{mg} / \mathrm{kg}$ de isoniazida $e$ $19 \mathrm{mg} / \mathrm{kg}$ de etambutol. A dose total média de estreptomicina foi de $81 \mathrm{~g}$ nos 47 pacientes com menos de 40 anos e de $71 \mathrm{~g}$ nos 31 restantes.

Definiu-se como eficácia teórica a taxa de cura de pacientes que inicialmente tinham baciloscopia positiva no escarro e que fizeram regularmente o tratamento, excluindo-se os abandonos, os óbitos por causa não tuberculosa e os pacientes cujos esquemas foram modificados pela substituição de um ou mais medicamentos. Os eventos finais em 59 pacientes bacilíferos podem ser vistos na Tabela 4. Observa-se que, dos 46 pacientes que fizeram uso regular da medicação, $41(89,2 \%)$ resultaram curados. Excluindo-se, desse subgrupo, os dois pacientes que abandonaram o tratamento e o caso de óbito que ocorreu por causa não tuberculosa, restaram 43 pacientes. Dessa forma, curaram-se $41 / 43$ pacientes baciliferos que utilizaram o esquema SHM regularmente até o final, resultando em eficácia teórica de 95,3\%.

O período de seguimento dos 41 pacientes bacilíferos curados, através do Sistema de Informações do Programa de Tuberculose, variou de cinco a mais de 10 anos. Não se verificaram recidivas.

Dentre os fatores observados, o mais significativo fator de risco para os pacientes não alcançarem a cura foi o uso irregular da medicação. Entre os pacientes que usaram regularmente a medicação, a taxa de cura foi de mais de $88 \%$, enquanto nos demais esse percentual não chegou a 38\% (Tabela 2) ( $p=0,0005)$. Na amostra examinada, as variáveis peso, idade, sexo, cor e doses dos medicamentos não influíram significativamente nos resultados.

\section{DisCUSSÃO}

Os dados foram obtidos na rotina de serviço de uma unidade ambulatorial de saúde, com tratamento auto-ad- 
ministrado. Sabe-se que essa estratégia predispõe à irregularidade de uso da medicação e ao abandono de tratamento ou falência, caso o serviço que presta assistência não esteja suficientemente organizado e/ou caso os pacientes apresentem problemas psicossociais ${ }^{(7,8)}$, como o alcoolismo. A importância dessa situação clínica como fator associado ao insucesso de tratamento também foi confirmada na presente série, na qual se observa que o uso irregular da medicação e a taxa de abandono foram significativamente maiores entre os alcoolistas. Assim, é devido ao alcoolismo que a exclusão dos pacientes com doença hepática crônica (Tabela 1) melhora os resultados, elevando a taxa de cura para 81,5\%.

Usando análises semelhantes, os esquemas RHM e RHZ, na mesma unidade de saúde ${ }^{(2)}$, resultaram em taxas de cura de $90,8 \%$ e $90,5 \%$ respectivamente, significativamente maiores $(p<0,001)$ (Tabela 5) que a obtida com o SHM. Esse pior resultado do SHM pode ser explicado pelo maior poder bactericida de esquemas contendo rifampicina, ou rifampicina e pirazinamida. Outra explicação seria que os pacientes que usaram SHM eram mais doentes, em função dos critérios de indicação. Nesse grupo, por exemplo, havia $10,2 \%$ de casos de tuberculose miliar, forma grave de tuberculose. Esse número supera em muito os $0,6 \%$ descritos na mesma unidade no período de 1983 a $1988^{(6)}$ e os 0,3 e 1,3\% referidos nas séries de Morrone et al.(9). Outro aspecto a considerar, que poderia explicar a diferença entre os resultados, diz respeito ao bom nível organizacional da UT3 na época dos tratamentos com RHM e RHZ, que permitia a obtenção de taxas de abandono tão baixas (Tabela 5). Faz-se essa colocação porque a partir de 1988 a taxa de abandono cresceu progressivamente na unidade, de modo que no final de 1994 tinha alcançado cerca de $18 \%(10)$. E 58 dentre os 78 pacientes desta coorte foram inscritos após essa data.

De qualquer modo, a taxa teórica de cura com o esquema SHM, de 95,3\%, não se diferencia significativamente

TABELA 5

Comparação dos eventos finais obtidos com os esquemas RHM (de $1 / 80$ a 3/82), RHZ (de $9 / 83$ a 12/88) e SHM (de $1 / 86$ a $12 / 94$ ), na UT3

\begin{tabular}{|c|c|c|c|c|c|c|}
\hline \multirow[t]{2}{*}{ Eventos finais } & \multicolumn{2}{|c|}{ RHM } & \multicolumn{2}{|c|}{ RHZ } & \multicolumn{2}{|c|}{ SHM } \\
\hline & $\mathbf{N}$ & $\%$ & $\mathbf{N}$ & $\%$ & $\mathbf{N}$ & $\%$ \\
\hline Cura* & 534 & 90,7 & 837 & 90,5 & 58 & 77,3 \\
\hline Abandono & 29 & 5,0 & 42 & 4,5 & 8 & 10,7 \\
\hline Falência & 9 & 1,5 & 33 & 3,6 & 5 & 6,7 \\
\hline Óbito & 16 & 2,6 & 13 & 1,4 & 4 & 5,3 \\
\hline Total & 588 & 100 & 925 & 100 & 75 & 100 \\
\hline
\end{tabular}

* Taxas de cura com RHM e RHZ significativamente maiores que a obtida com SHM ( $p<$ $0,001)$. da taxa obtida na mesma unidade com o esquema RHZ, que foi de $97,8 \%{ }^{(11)}$.

Outro aspecto a ser discutido diz respeito à tolerabilidade. Embora a incidência de efeitos adversos tenha sido elevada (41\%), somente em $10,2 \%$ dos pacientes foi necessário proceder a adaptações no esquema, sendo necessária a troca de um ou mais fármacos em 3,8\%. Essa taxa de troca do esquema foi semelhante à observada nos 955 pacientes tratados com RHZ na UT3, que foi de 3,1\%(2). Castelo et al. relataram taxa de apenas 1\% com esse último esquema ${ }^{(12)}$. Já o British Medical Research Council referiu $2,8 \%$ de troca quando o esquema era $\mathrm{SRH}$ (estreptomicina, rifampicina e etambutol)(13). No entanto, Bobrowitz refere um valor bem mais alto, de $21,1 \%$, com o esquema SHM; embora esse autor tenha utilizado $450 \mathrm{mg} /$ dia de isoniazida, o fármaco responsável pela maioria dos efeitos adversos foi a estreptomicina, com taxa de 18,3\%, sendo que, em 9,6\% dos casos, devido à ototoxicidade ${ }^{(14)}$.

Uma explicação possível para a elevada taxa de vertigem $(24,4 \%)$ seria que os pacientes com o efeito adverso receberam dose total mais alta de estreptomicina. No presente estudo a dose total média de estreptomicina utilizada foi $81 \mathrm{~g}$ nos pacientes com 40 ou menos anos e $71 \mathrm{~g}$ naqueles com mais de 40 anos. Portanto, o grupo de mais idade recebeu dose maior que a recomendada, que era de, no máximo 67,5g. Embora a taxa de vertigem tenha sido maior nesses pacientes (29\%) do que nos com menos idade $(21,3 \%)$, a diferença não foi significativa. Também não foi significante a necessidade de modificação do esquema inicial por vertigem $(9,7 \%$ contra $8,5 \%$ nos pacientes mais jovens). Acrescenta-se que as três trocas de esquema verificaram-se nos pacientes do grupo de menos idade. Isso parece sugerir que a sensibilidade individual ao fármaco pode ser tão importante quanto a dose. Suspeita-se hoje da existência de um componente genético que torna alguns indivíduos mais suscetíveis aos efeitos ototóxicos dos aminoglicosídeos ${ }^{(15)}$. Porém, na prática médica diária, ainda não se pode prever qual paciente terá dano otológico.

A taxa de hepatotoxicidade pelo esquema SHM $(1,3 \%)$ foi semelhante à de Bobrowitz, que obteve um valor de 1,9\% em 104 pacientes tratados com esses três tuberculostáticos, porém com doses mais altas de isoniazida e etambutol (14). Por outro lado, foi bem menor do que os 3,5\% obtidos no Hospital Sanatório Partenon no estudo realizado de 1984 a 1986, em 57 pacientes sem tratamento prévio, portanto, sem hepatopatia medicamento$\mathrm{sa}^{(5)}$. Na presente série, entre os 45 casos de indicação do SHM devido à ocorrência de lesão hepática no tratamento inicial com RHZ, observou-se apenas uma recidiva da hepatopatia (2,2\%). Esses dados sugerem que o esquema SHM pode ser mais uma alternativa para os casos de hepatotoxicidade pelo RHZ, situação para a qual não existe 
um esquema padronizado. No I Consenso Brasileiro de Tuberculose $^{(16)}$ não se incluiu o esquema SHM entre as alternativas para os casos de hepatopatia medicamentosa pelo RHZ.

Conclui-se que o esquema SHM tem boa tolerabilidade, pois somente $3,8 \%$ dos casos tiveram o esquema trocado; razoável eficiência, pois a taxa geral de cura foi de 77,3\%; e excelente eficácia, com taxa teórica de cura de 95,3\%. Por isso, na experiência dos autores, é um esquema adequado para uso nas situações clínicas nas quais não se recomenda o RHZ. Desde 2001, o SHM foi incorporado à Norma Técnica da Política de Controle da Tuberculose do Estado do Rio Grande do Sul(17), para ser utilizado nas situações de risco referidas, sendo essa conduta reservada para pacientes internados em hospitais de referência, sob a orientação de supervisores do programa. No entanto, essa conduta não é consenso entre os pneumologistas e não está contemplada nas normas do Ministério da Saúde ${ }^{(18)}$.

As doses da estreptomicina devem ajustar-se ao peso e à idade dos pacientes, que devem ser vigiados para a detecção de sinais clínicos precoces de comprometimento do oitavo par craniano, principalmente de seu ramo vestibular. Pode-se, ainda, cogitar o uso de substâncias protetoras contra a ação nefasta do aminoglicosídeo, conforme se tem preconizado mais recentemente $e^{(19)}$. Finalmente, recomenda-se atenção especial aos casos de alcoolismo associado, pela grande freqüencia de uso irregular dos medicamentos e insucesso do tratamento.

\section{REFERÊNCIAS}

1. Fraga H, Sefton AJ, Gerhardt, G. Controle do tratamento da tuberculose pulmonar em áreas urbanas do Brasil. Rev Serv Nac Tuberc 1969; 13:329-49.

2. Picon PD, Rizzon CFC, Freitas TM. Resultados do tratamento. In: Picon PD, Rizzon CFC, Ott WP, editors. Tuberculose: epidemiologia, diagnóstico e tratamento em clínica e saúde pública. Rio de Janeiro: MEDSI, 1993;506-23.

3. Fox W. Estado atual de la quimioterapia acortada de la tuberculosis. Bol Union Int Contra Tuberc 1978;53:278-91.
4. Chaulet P. La quimioterapia de la tuberculosis en 1983. Bol Union Int Contra Tuberc 1983;58:26-36.

5. Picon PD, Espina CAA, Rizzon CFC. Efeitos adversos aos esquemas de tuberculostáticos. In: Picon PD, Rizzon CFC, Ott WP, editors. Tuberculose: epidemiologia, diagnóstico e tratamento em clínica e saúde pública. Rio de Janeiro: MEDSI, 1993;531-43.

6. Picon PD, Rizzon CFC, Picon MIX, Souza ALS. Hepatopatia aos tuberculostáticos. In: Picon PD, Rizzon CFC, Ott WP, editors. Tuberculose: epidemiologia, diagnóstico e tratamento em clínica e saúde pública. Rio de Janeiro: MEDSI, 1993;543-59.

7. Teixeira GM. O desafio de segurar sob tratamento até a cura o paciente de tuberculose. Bol Pneumol Sanit 1995;3:3-9.

8. Natal S, Valente J, Gerhardt G, Penna ML. Modelo de predição para o abandono do tratamento da tuberculose pulmonar. Bol Pneumol Sanit 1999;7:65-78.

9. Morrone N, Solha MSS, Cruvinel MC. Tuberculose: tratamento supervisionado "versus" tratamento auto-administrado. J Pneumol 1999;25: 198-206.

10. Osório JF, Figueiredo E, Freitas TM, Maciel D, Bender D, Silveira YC, et al. Tratamento ambulatorial auto-administrado e com supervisão semanal, aplicado a pacientes egressos de um hospital de tuberculose. Bol Saúde. In press 2002.

11. Picon PD, Della Giustina ML, Janczewski CA. Tratamento da tuberculose com o esquema RHZ. J Pneumol 2000;26(Supl 3):S19.

12. Castelo A, Jardim JRB, Goihman S, Kalckman AS, Dalboni MA, Silva EA, et al. Comparison of daily and twice-weekly regimens to treat pulmonary tuberculosis. Lancet 1989;2(8673):1173-6.

13. East African/British Medical Research Council Study. Results at 5 years of a controlled comparison of a 6-month and a standard 18-month regimen of chemotherapy for pulmonary tuberculosis. Am Rev Respir Dis $1977 ; 116: 3-8$.

14. Bobrowitz ID. Ethambutol-Isoniazid versus Streptomycin-EthambutolIsoniazid in original treatment of cavitary tuberculosis. Am Rev Respir Dis $1974 ; 109: 548-53$.

15. Fischel-Ghodsian N, Prezant TR, Chaltraw WE. Mitochondrial gene mutation is a significant predisposing factor in aminoglycoside ototoxicity. Am J Otolaryngol 1997;18:173-8.

16. I Consenso Brasileiro de Tuberculose. Efeitos colaterais e interações medicamentosas. J Pneumol 1997;23:305-10.

17. Rio Grande do Sul. Secretaria Estadual da Saúde. Norma Técnica da Política de Controle da Tuberculose. Porto Alegre, 2001.

18. Brasil. Ministério da Saúde e Funasa. Plano Nacional de Controle da Tuberculose. Normas Técnicas. Estrutura e Operacionalização. Brasília, 2000.

19. Song B-B, Sha S-H, Schacht J. Iron chelators protect from aminoglycoside-induced cochleo-and vestibulo-toxicity. Free Radic Biol Med 1998;25:1-7. 